\title{
Cutaneous manifestation mimicking Stevens- Johnson syndrome in a critically ill patient: looks similar but totally different
}

\author{
Jo A Lim, SE Chong *, Huda Zainal Abidin, Mohd H Hassan
}

Hong Kong Med J 2017;23:653.e3-4

DOI: 10.12809/hkmj165051

\section{Case}

A 60-year-old gentleman was intubated due to severe leptospirosis and multi-organ failure: acute kidney injury and liver failure with disseminated intravascular coagulation (DIC). He was ventilated in the intensive care unit for 7 days. He was treated with ceftriaxone, pantoprazole, fentanyl, midazolam, and required frequent fluid challenge and inotropic support.

On the day of extubation, the patient was noted to have ulcers over the angles of the mouth with crusted blood and seropurulent discharge (Fig 1). He also had diffuse erythema and desquamation over the tips of the fingers (Fig 2), and a large purpuric patch over the lateral aspect of both thighs (Fig 3) with generalised scaly dry skin over the body.

He was treated as Stevens-Johnson syndrome (SJS). Antibiotic therapy was stopped and intravenous hydrocortisone was started but his ulcers continued to worsen. A dermatological opinion was arranged and revealed that the oral and tongue mucosa erosions were confined to the site of previous endotracheal tube placement rather than being the diffuse oral and lips erosions of SJS. Nikolsky sign was negative. The conjunctiva was clear, and there was involvement of the nasal, urethral or anal mucosa.

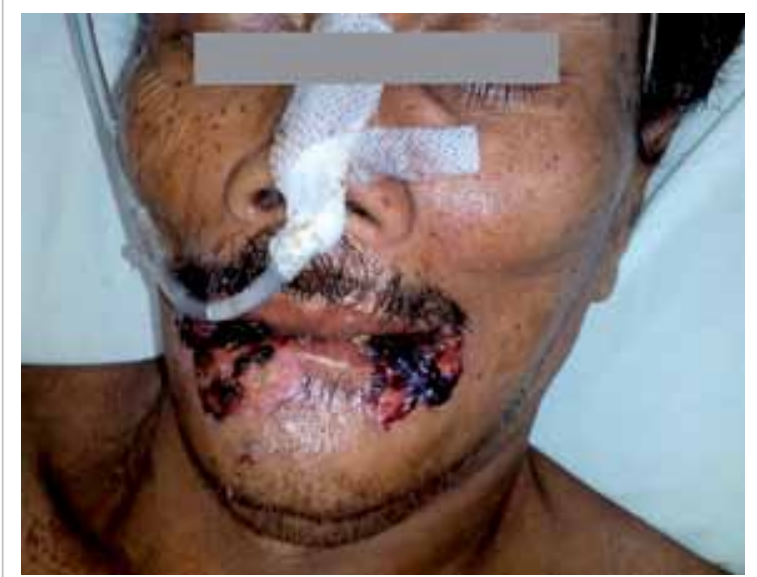

FIG I. Localised oral and lip ulcers confined to previous endotracheal tube placement site, as opposed to the diffuse oral and lip ulcers seen in Stevens-Johnson syndrome
In view of the confined area of mucosa involvement, he was diagnosed with medical devicerelated pressure ulcers. The purpura and ecchymosis were due to the underlying coagulopathy secondary to septic shock with DIC. Potential infective causes, eg vegetating herpes simplex, staphylococcal scalded skin syndrome, were excluded by negative wound culture. There were also no features of SJS on skin biopsy.

The steroid was stopped immediately and antibiotics resumed. Albumin level was optimised. After 2 weeks of oral care, the patient's skin condition improved (Fig 4) and he finally attained full recovery.

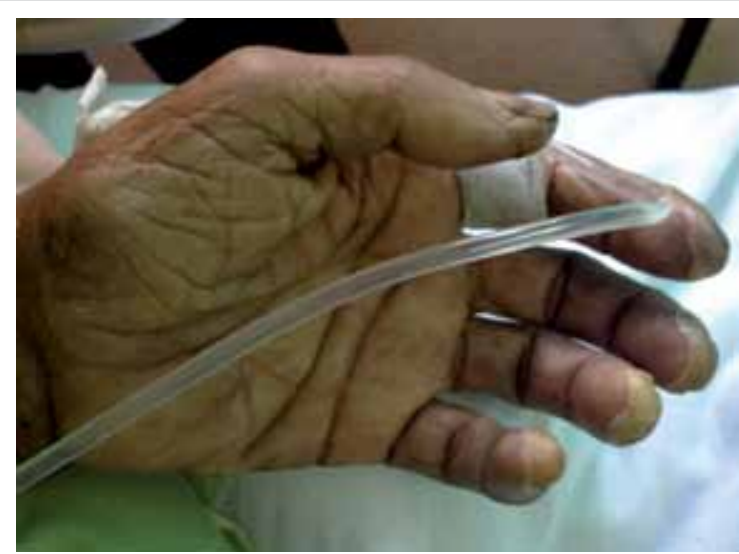

FIG 2. Erythematous fingers with skin desquamation over the fingertips

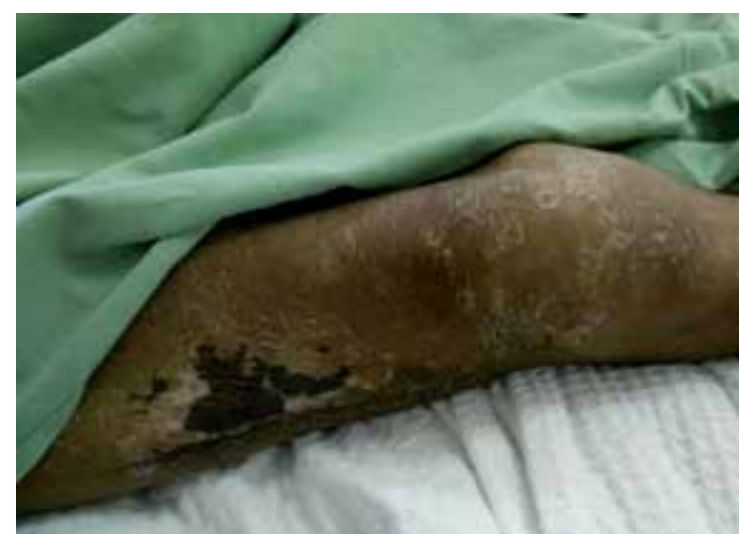

FIG 3. Purpuric patch with scaly skin rash over lateral thigh 


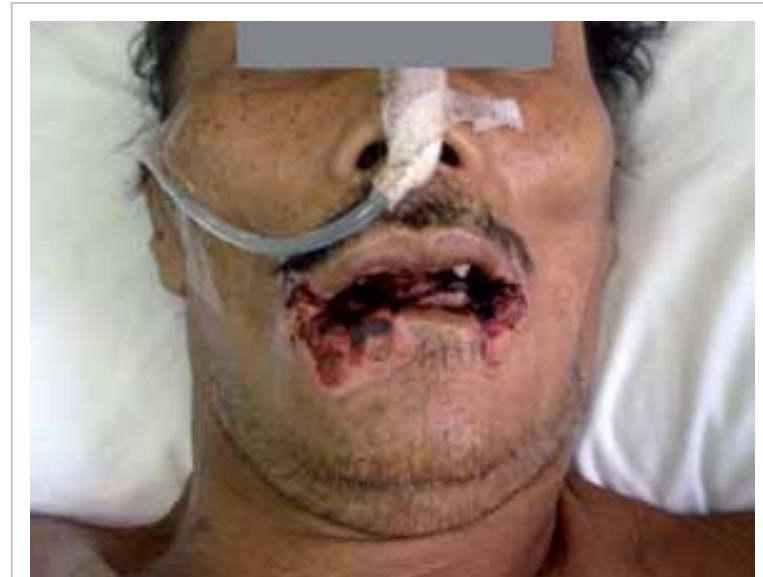

FIG 4. Ulcers improved 4 days after stopping hydrocortisone and restarting antibiotics

\section{Discussion}

Tracheal intubation is one of the best methods of airway protection ${ }^{1}$ but serious complications may occur, especially in critically ill patients. ${ }^{2}$

Stevens-Johnson syndrome is part of a spectrum of severe cutaneous adverse reactions that affect skin and mucous membranes. It is commonly caused by certain medications or infections. Skin lesions may be purpuric macule spots, erythema, or sometimes violaceous target-like lesions. Mucous membrane erosions and ulcers almost always appear in the eyes, mouth and lips, but may also occur in the upper airway, genitourinary and gastrointestinal tract. Assessment is often difficult when mucous membrane involvement is not yet apparent. ${ }^{3}$

Hypotensive episodes during septic shock may lead to reduced perfusion pressure to the skin and mucosa. This may cause pressure point areas to develop pressure sores. ${ }^{4}$ Hypoalbuminaemia and DIC may cause further deterioration of the ulcers. Pressure sores are one of the most common complications among patients undergoing mechanical ventilation in a poorly managed setting. ${ }^{5}$
Physicians should always be extra cautious when diagnosing SJS. The consensus definition should always be consulted with referral to a dermatologist or performance of a skin biopsy if there is any doubt. ${ }^{3}$ An incorrect diagnosis of SJS may lead to a totally inappropriate spectrum of treatment such as cessation of appropriate lifesaving antibiotics and the commencement of steroid therapy that will increase the risk of infection and impair wound healing.

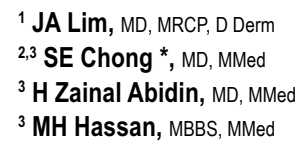

1 Department of Internal Medicine, Hospital Sultan Abdul Halim, 08000 Sungai Petani, Kedah, Malaysia

${ }^{2}$ Regenerative Medicine Cluster, Advanced Medical and Dental Institute, Universiti Sains Malaysia, Bertam, 13200 Kepala Batas, Penang, Malaysia

${ }^{3}$ Department of Anesthesiology and Intensive Care, School of Medical Sciences, Universiti Sains Malaysia, 16150 Kota Bharu, Kelantan, Malaysia

* Corresponding author: sechong@usm.my

\section{References}

1. International Liaison Committee on Resuscitation. 2005 International Consensus on Cardiopulmonary Resuscitation and Emergency Cardiovascular Care Science with Treatment Recommendations. Part 4: Advanced life support. Resuscitation 2005;67:213-47.

2. von Goedecke A, Herff H, Paal P, Dörges V, Wenzel V. Field airway management disasters. Anesth Analg 2007;104:481-3.

3. Mockenhaupt M. Severe drug-induced skin reactions: clinical pattern, diagnostics and therapy. J Dtsch Dermatol Ges 2009;7:142-60.

4. Black JM, Edsberg LE, Baharestani MM, et al. Pressure ulcers: avoidable or unavoidable? Results of the National Pressure Ulcer Advisory Panel Consensus Conference. Ostomy Wound Manage 2011;57:24-37.

5. Tang WM, Tong CK, Yu WC, Tong KL, Buckley TA. Outcome of adult critically ill patients mechanically ventilated on general medical wards. Hong Kong Med J 2012;18:284-90. 